\title{
Go Slow to Go Fast: Successful Engagement Strategies for Patient-Centered, Multi-Site Research, Involving Academic and Community-Based Organizations
}

\author{
Laura T. Pinsoneault, Ph.D. ${ }^{1}$, Emily R. Connors, M.S. ${ }^{2}$, Elizabeth A. Jacobs, M.D. ${ }^{3}$, and \\ Jerica Broeckling, M.S. ${ }^{4}$
}

${ }^{1}$ Spark Policy Institute, Denver, CO, USA; ${ }^{2}$ Clinical and Translational Science Institute, Medical College of Wisconsin, Milwaukee, WI, USA; ${ }^{3}$ Dell Medical School, University of Texas at Austin, Austin, TX, USA; ${ }^{4}$ Alliance for Strong Families and Communities, Milwaukee, WI, USA.

BACKGROUND: In 2010, the Patient Centered Outcomes Research Institute (PCORI) was created to fund patientcentered research that meaningfully engages stakeholders impacted by that research. As a result, investigators became interested in understanding who are appropriate stakeholders and what meaningful engagement in research looks like (6, 8-10).

OBJECTIVE: To understand how and when stakeholder engagement worked well and identify areas for enhancing engagement in a PCORI-funded research study of peer-topeer support of older adults in three communities across the USA.

DESIGN: Qualitative interview study.

PARTICIPANTS: Twelve members of the inter-disciplinary research team.

APPROACH: Interviews were conducted via phone, recorded, and transcribed. Transcripts were analyzed using a constant comparative method to identify themes. Transcripts were independently coded; coded themes were discussed by a small group of the research team to check interpretation and clarify meaning. Once initial themes were identified, the interviews and codes were shared with an external consultant who recoded all 12 transcripts and conducted further analysis and interpretation. Documentation from research meetings was used to validate our findings.

KEY RESULTS: Strategies for facilitating meaningful engagement in the partnership, proposal, study design, and planning phase were very similar to community-based participatory research and include the use of community to identify research needs, equitable compensation and leadership, and budgeting for engagement activities. Strategies in the data collection phase include the use of cultural brokers, weekly data calls between the academic PI and imbedded research assistants, and maintaining joint ownership for research.

CONCLUSIONS: Major funding institutions (e.g., NIH, PCORI) recognize that community engagement leads to higher quality, more meaningful research $(7,21)$. Our results support that assumption and in addition, suggest

Electronic supplementary material The online version of this article (https://doi.org/10.1007/s11606-018-4701-6) contains supplementary material, which is available to authorized users.

Received March 7, 2018

Revised July 25, 2018

Accepted October 3, 2018

Published online October 23, 2018 an investment in engagement strategies at the onset of a research project and the use of cultural brokers can greatly contribute to the success of implementing a large, multi-site research project.

KEY WORDS: patient-centered outcomes research; qualitative research; community-based participatory research; aging; evaluation.

J Gen Intern Med 34(1):125-31

DOI: $10.1007 / \mathrm{s} 11606-018-4701-6$

(C) Society of General Internal Medicine 2018

\section{INTRODUCTION}

In 2010, the US Congress authorized the creation of the Patient Centered Outcomes Research Institute (PCORI) as part of the Affordable Care Act. PCORI's mission is to fund patient-centered research that meaningfully engages stakeholders who will be impacted by the results of that research. ${ }^{1}$ Public health practitioners applauded this development as they long recognized involving community and collaborating with patients and community stakeholders is essential in efforts to improve public health; community engagement has been a center point of public health programs that have successfully addressed health issues such as smoking cessation, obesity, and heart disease. ${ }^{2-5}$ As the complexity and urgency of health problems continue to increase, stakeholder engagement is being recognized as vital to providing quality care, preventing disease, effectively translating research findings into practice, and key to achieving health equity. ${ }^{6-8}$ However, this was a new model and approach to research for many investigators, as they were not sure who were appropriate stakeholders and what meaningful engagement in healthcare research looks like. ${ }^{6,8-10}$ The clinical effectiveness research (CER) field has only recently begun to explore the use of communitybased participatory research (CBPR) methods and partnerships to increase stakeholder engagement. ${ }^{11}$

PCORI defines engagement in research as "the meaningful involvement of patients, caregivers, clinicians, and other healthcare stakeholders through the research process - from topic selection through design and conduct of research to dissemination of results". ${ }^{12}$ Meaningful stakeholder engagement in research lays the foundation for the validity, 
accelerated use, and sustainability of findings to improve health. ${ }^{13}$ It ensures that research is grounded in experience, increasing the value of the results to community and researchscientists. ${ }^{9}, 14,15$

To help provide guidance to other investigators on promising stakeholder engagement practices, we interviewed a PCORI-funded team of investigators and stakeholders to understand how and when engagement worked well, and to identify how to enhance that engagement.

\section{METHODS}

\section{Participants and Setting}

The Alliance for Strong Families and Communities (Alliance), which represents and supports a network of more than 400 community-based organizations (CBOs) in the USA, sought out an investigator interested in working with its members to conduct research on the role of CBOs in improving health outcomes in their communities. This led to a partnership between the Alliance and Dr. Elizabeth Jacobs who, at the time, was a professor of medicine and population health at the University of Wisconsin at Madison. Dr. Jacobs had experience working with communities using a CBPR approach and valued the expertise and insight community partners/ stakeholders provide to research. Together, they surveyed Alliance member organizations to identify communitybased interventions that, at face-value, had an impact on the health of community members but lacked empirical evidence. In this process, we identified peer-to-peer support to promote aging in place as an important patientcentered intervention, as a focus of our research, and partnered with three organizations that provided these services: Alpert Jewish Family and Children's Service of West Palm Beach, the Community Place of Greater Rochester, and Jewish Family Service of Los Angeles. We were funded by PCORI in 2014 to conduct a three-year study in which we compared the effectiveness of peer-to-peer support vs. usual community services in helping older adults age well in their communities. ${ }^{16,17}$ Due to the team's commitment to stakeholder engagement, we embedded several engagement strategies commonly used in CBPR to ensure that community members would have meaningful participation.

We formed a research team of 24 people from academia, the Alliance, leadership of the CBOs, program directors of aging services, and representatives from each community of older adults receiving peer services, caregivers of older adults providing peer services, and community members who have a passion for promoting health in their community. At the start of the study, we came together to talk about what meaningful engagement would look like and agreed that it results from having agreed-upon shared goals, a willingness to push boundaries, mutual respect, and equitable partnerships.

\section{Data Collection}

One and a half years into the three-year peer-to-peer research project, the Associate Director of the Alliance's Center for Engagement and Neighborhood Building conducted 12 qualitative one-on-one phone interviews with members of the research team to better understand where and how we succeeded and failed at creating and maintaining meaningful engagement across state lines and time zones. The interviewer used a semi-structured interview guide designed to elicit information about how the project design activated or hindered meaningful engagement across the various roles of the research team and where we could improve engagement. Interviews were recorded and transcribed. The study was deemed exempt from IRB review by the University of Wisconsin Health Sciences IRB.

\section{Data Analysis}

The interview transcripts were analyzed by an Alliance project staff and two Alliance non-project staff using constant comparative method of coding and analyzed for themes. ${ }^{18}$ Transcripts were independently coded; coded themes were discussed by a small group of the research team to check interpretation and clarify meaning. Once initial themes were identified, the interviews and codes were shared with an external consultant outside of the project team, who could provide a level of independence and reduce bias in interpreting results, who recoded all 12 transcripts and conducted further analysis and interpretation. Documentation from research team meetings was used to further validate and develop primary findings. Participants included representatives from each of the five partnering organizations and all identified roles: three research assistants, three community stakeholders, three representatives from the leadership of the CBOs (site PIs), two overall investigators, and a representative from the statistician team.

\section{RESULTS}

Our respondents talked about engagement and how it differed in two different phases of the research: the partnership, proposal, study design and planning phase, and the participant enrollment and data collection phase.

\section{Strategies That Facilitate Meaningful Engagement in the Partnership, Proposal, Study Design, and Planning Phase}

Meaningful engagement started with the development of the research team's relationship, in how we chose the research topic, and in the budgeting for engagement activities, including compensation of stakeholders.

Use of Community to Identify Research Needs. The focus of the research on peer-to-peer support was a product of our deliberate engagement process. We surveyed CBOs to identify 
common community initiatives that they believed positively influenced health. Stakeholder input on the survey served as the source for the identification of the population of interest, the promising initiatives being implemented, and the communities where engagement practices were actively being used to promote outcomes. Further refinement of the research focus and design evolved from interviews with CEOs, clustered around work with older adults implementing peer-to-peer support to determine whether enough commonalities existed in implementation to meet the criteria for a comparative effectiveness trial. The interviews helped identify and shape the research questions. Once selected, work began with the communities input to develop the full PCORI application including the research design and the PCORI required engagement rubric. ${ }^{19}$

Equitable Compensation and Leadership. Key leaders within each organization (e.g., CEO, VPs) were included in the leadership team of the peer-to-peer research study as site-PIs and compensated for their expertise and substantial contributions. Their budgets also included funds to hire an embedded researcher employed by the local $\mathrm{CBO}$, rather than the university. The academic PI approved the hire and directed research responsibilities, but this position was employed by and supervised by the $\mathrm{CBO}$. This arrangement provided the substantial support needed for CBOs to partner in research, as nonprofits often operate with very little margin in their budgets, and have few dollars to allocate to ongoing research and evaluation, even when they are not in the lead. Team members from the community conveyed the importance of equitable compensation for the work needed to support research activities in their organization when we interviewed them about engagement practices:

For community organizations and researchers too, there is an actual financial cost to have a community organization involved in a research project. As researchers are designing these projects, remember to support the collaborating organizations financially. Whether that be cash resources or other resources ..., having the research assistants fullyfunded by the research project [ensures] they are compensated for it.Embedded researchers also brought valuable research knowledge to CBOs. They were responsible for the day-to-day work of research implementation related to participant recruitment, longitudinal data collection, and troubleshooting. They could also answer staff questions and respond to concerns around how the research process would impact service, a core concern of CBOs when they get involved in research.

In each of the communities, two stakeholders were employed as paid members of the research team with full participation in all research meetings and activities. These stakeholders provided important input into the day-to-day lives of older adults and their experiences which helped inform the research instruments and recruitment strategies.

Budgeting for Engagement. The PCORI Engagement Plan included financial resources for engagement activities to support critical research tasks from design to dissemination, including annual face-to-face meetings and community site visits for the full team. Regularly scheduled calls and community engagement learning opportunities that did not include discussions of the research protocol were also planned with the purpose of monitoring and engagement of stakeholders throughout the length of the project. These practices established rapport and the value of the stakeholders in the research process, but also built team culture and trust, and ultimately, improved the conduct of the study. Engagement activities were led by a "cultural broker" from the Alliance, who has deep expertise in developing community engagement.

\section{Strategies That Facilitate Meaningful Engagement During the Research}

Three additional strategies promoted meaningful engagement once the peer-to-peer research project was funded, including the active use of cultural brokers, weekly data calls between the academic PI and embedded research assistants, and maintaining joint ownership of the research.

Active Use of Cultural Brokers. Cultural brokering is defined as "bridging, linking, or mediating between groups or persons of different cultural backgrounds to effect change". ${ }^{20}$ The peer-to-peer research project employed the use of a "cultural broker" - the Alliance-who served as a bridge between the academic partner and the CBOs. This role was twofold ${ }^{1}$ : to serve as a "translator" to help the academic researchers and community-based practitioners understand each other and their different assumptions and values they brought to the project; and ${ }^{2}$ to monitor best practices around engagement, serving as a professional development coach for the full team to further bolster the effectiveness of what can sometimes be competing goals. As one team member said:

Cultural brokering is the right term; there are different ways that each sector speaks. Understanding the day-today of both sectors, and in both research and practice, has been valuable.This role was key to meaningful engagement throughout the peer-to-peer research project because the research involved multiple institutions, each with their own communication style, culture, geographic nuances, and perspectives of community-engaged, patient-centered research. In the first 18 months, building shared language between academic research and community-based practice was key, allowing both sides to assert their individual goals, as well as shared goals for the peer-to-peer research project. 
For one site, the use of a cultural broker was essential to their participation. As this site PI indicated, when we interviewed them about engagement practices, they likely would not have participated in such an opportunity if it had not been for the use of this cultural broker:

I don't think I would have engaged in this kind of research project with a university like Wisconsin, or that matter, any university without the Alliance acting as the third part of the stool. As much as I would have wanted to get the data at the end of the study, I am not sure that we, as social service agencies belong in the world of medical studies. I'm just not sure that is our bailiwick and I think because of the application of engagement, this changed everything for me. Now we are going to be able to be engaged in applying the peer relationship piece and get a lot out of the project for ourselves.

In the engagement interviews, team members emphasized the importance of co-defining and agreeing on roles and responsibilities up front. Roles were spelled out in the proposal stage but defined again at the outset of the project, sometimes redefined through staff changes or for other reasons throughout the project-a challenge alerted to us through the interview process. The intentional exercise of re-defining roles as a group through neutral facilitation by a cultural broker meant each member could identify ways they could contribute their expertise and experience, bring to light other assumptions and expectations, and create a pathway for input and engagement rather than being assigned a role, further contributing to a positive team culture. This process, facilitated by the cultural broker, reinforced the distribution of power among the team members through shared understanding of the value each member brought to the table.

Alignment and building a common understanding of the value of "engagement" in the research project and goals provided a foundation for preventing and addressing challenges that can arise in a large, multi-site research project:

We learn a lot from one another. We're all working from the same purpose, and we are all there to share our experiences and that engagement is really important [to] creating a real great team.

The cultural broker was used as a sounding board for both stakeholder groups, which allowed the team to be more proactive rather than reactive. This was one way in which the cultural broker monitored engagement practices and served as "coach" for the research team. As one participant explained through the engagement interviews:
Their involvement in this project has been really valuable because I do really see them as a cultural broker for helping communities understand the research side and for helping me understand how to work with this particular social service agency. I've worked with communities before, but not with agencies like this and really understanding how they work had been helpful for me.

The cultural broker, was in part, responsible for ensuring meaningful engagement happened and that there was a plan in place to address any issues that may arise. Another challenge brought to our attention through the engagement interviews was how well the sites were engaging the older adult stakeholders in the research project; once the study was up and running and input had been received and incorporated into the design and implementation of the project, sites did not know how to engage the older adults further in the research project. The team developed a plan for when and how to communicate when conflict and problems, such as this one, arose during the research project, regardless of the type of problem. This extended beyond just who had the authority to make decisions, but how those decisions were going to be made, who was going to be included in those decisions, and what forms of communication were acceptable to those involved for addressing those decisions. The cultural broker held calls which included some discussions without the academic members of the team to explore the balance of power in the research process. Issues that surfaced during these calls, with the support of the cultural broker, could then be brought to larger team meetings for discussion with the full research team. The cultural broker provided translation and intervention when interpersonal conflict and miscommunication occurred, as this was most often a result of external pressures coming from within one's own discipline or sector that were not understood by other parties:

I feel like I can come to the cultural broker and the CBOs can also come to the cultural broker at different points and say, 'I'm worried about this, how should I approach the academic partner?' or I can go to them and ask how I should approach the CBO. Having that touchstone to think about how to do that has been really valuable and created a space and an avenue for actually thinking about the problem before acting on addressing it, which has made us more proactive than reactive.

Weekly Data Calls Between the PI and the Embedded Researchers at the CBOs. Weekly data calls were held between the academic PI and the embedded research assistants. The research team cited the importance of these 
weekly data calls to bring forth and address questions related to recruitment goals, data collection experiences, data monitoring, and general updates, among others, when we interviewed them about engagement practices:

The weekly calls are a really good place for others to get an update, see how everybody is doing, but also a place to bounce off ideas or questions, as the team can work through it together, which is great... this has been a smooth process from the start really - the open communication and willingness to help each other.

These calls also provided context for unique community challenges that could be discussed and problem-solved as a group either on that call or via other calls held with the larger research team. Weekly data calls were also a way to build the notion of "team," provide reassurance to the PI and others that the research was progressing at an acceptable rate, or if not, allow for problem-solving between the PI and sites. The calls facilitated the flow of information on how to adapt or change research protocols and processes to allow for better data to be collected. Given the national, multi-site team structure, intentional efforts to build the team and provide training through regular calls, in addition to in-person meetings, contributed to the success of the project by building trust and strong working relationships across long distances:

Face-to-face meetings are very important, in addition to the regular calls. This really builds community.

Maintain Joint Ownership for Research. Using researchers employed at the CBO fostered joint ownership by the CBO and academic partner of the research process. This offered community influence over implementation of data collection informed by needs of program participants and treatment of participants in the study. It also benefited the research, specifically working to answer the question for potential study participants, why would participation in research be meaningful to me? This practice was identified by the academic partner as a unique aspect of the research that made the study better:

...This is a really unique setup and it benefitted the study design, the recruitment and data collection... many of the sites were experts in recruiting patients and experts in running programs so what ended up happening was UW being able to get quality data and the sites being able to recruit patients [who needed peer to peer support].Embedded research staff at the CBO helped ensure the research progressed, in part due to the weekly data calls between the academic PIs and the community- based team, but also since they were physically on-site:

Having people onsite is definitely beneficial. I think the other [research] projects we have worked on; no one was onsite. It was done out of the university, and there were issues with even getting information back and forth because of HIPPA; things have to be picked up and dropped off, they couldn't be mailed. So, having people right on site has been extremely beneficial.

Researchers hired by the $\mathrm{CBO}$ put research as a real practice within organizations. Research assistants challenged existing perceptions that research gets done "to" community at the expense of the $\mathrm{CBO}$ and their mission. $\mathrm{CBOs}$ modeled this strategy as they implemented the research project within and throughout their organizations, as one team member described:

We have made a point to meet with [older adult] stakeholders on a regular basis to let them know what is going on and allowing them to ask questions [about the study].

This strategy also leverages the cultural capital and relationships of CBOs to engage and recruit members of the community who otherwise might not engage in research:

... [This] recognizes and is respectful of the stature that these organizations have in their community. In respecting this, we are successful in recruitment and collaboration.

The leaders of the CBOs indicated they would not engage in "traditional" research, if there was no respect for the CBO, their mission, and their expertise:

... The only way I could justify participating in a project like this - what was important to me was that we not get involved in something that was presented as 'here it is, roll it out. Do it and don't ask questions'. We should be protective of our clients, although there is an IRB, I really need to make sure that we were going to be getting involved with something that was really, as an organization, something that had checks and balances.

The benefits of community ownership extended beyond the data collection. The academic researcher recognized benefits to engaging a diverse research team and releasing some control of the research processes so that the results of the research could be better translated and used in the broader field of healthcare research: 
The purpose of stakeholder engagement is to make the research as successful as possible by really understanding the need and issues of older adults and their caregivers in the community, and therefore, [the stakeholders] contribute to the design, provide original ideas, but also problem-solve in helping us understand how we can do what we're doing from a research standpoint, even better so that we have more meaningful research and results.Finally, an often overstated but under practiced concept that fosters ownership is communication. Research team members interviewed could not understate the value of good, frequent communication. Good communication takes time, as one team member put it-"go slow, to go fast"; investing time up front in regular, transparent communication can payoff in the long term. This structure did not only benefit the research goals, but also increased the research capacity at the community level.

\section{DISCUSSION}

With influential institutions like PCORI and the NIH recognizing how stakeholder engagement in research can lead to better solutions for complex health problems, achieving the type of team structure and engagement we described is becoming an important ideal. ${ }^{7,21}$ The research team did not sacrifice the rigor of research or the meaningful engagement of stakeholders, but rather found a way for the two to complement each other and extend the benefits of the knowledge and learning process, as other PCORI-funded teams have found. ${ }^{22,23}$

Results from our engagement interviews suggest that meaningful engagement was kept front and center through all aspects of the peer-to-peer research project from how the project was initiated, designed, and proposed through the conduct and successful completion of the study. The research team was intentional about engagement and what meaningful engagement meant to each stakeholder group and member, another common theme across engagement research. ${ }^{20}$ The focus and intentionality around engagement through a cultural broker created a research model that was mutually beneficial to CBOs and academic researchers, an approach not well documented in engagement strategies for research in the literature. Both groups saw benefit in the data collected, CBOs had a desire to have "hard data" as evidence to the impact of their programs, the academic researcher sought answers to the research questions, and both the academic researcher and the $\mathrm{CBO}$ had a desire to understand what interventions allow older adults to age in place longer. Most, if not all, team members were predisposed to community-engaged research; therefore, there was a willingness and openness to learn about what works for certain stakeholders and a flexibility to change aspects of the research design, process, and strategies. In this case, the facilitation by the cultural broker, and the identification of a shared goal around engagement and what engagement means to the team, was a driving force that contributed to the success in implementing the peer-to-peer research project.

Our findings demonstrate how CBPR principles can be successfully applied in other research contexts and that they add to the success of other types of research designs. Our findings suggest that researchers and stakeholders need to invest in engagement strategies upfront- " go slow to go fast" and that the investment in the use of neutral cultural brokers for large, multi-site research projects can enhance the research. In this case, the cultural broker facilitated the identification of challenges or issues experienced by the team, individually and collectively, and aided in communication and problem-solving. Our interviews revealed that although roles for team members had been clearly defined at the outset of the project, after 18 months, there had been shifts in personnel and processes that necessitated a re-examining of roles. It also became clear that community stakeholders had an uneven experience across sites in the level of their engagement during various stages of the peer-to-peer research project. Having a cultural broker made it possible to work with the site leads to develop a set of common guidelines (see online appendices) for stakeholder engagement, which were then reviewed by, and shared with the full research team without slowing or interfering with the implementation of the research.

While this study provides us with insights into how CBPR principals can be applied in other research contexts, limitations do exist. The intent of the project was process improvement, specifically around engagement. The choice in interviewer, a member of the research team, and the director of engagement had the potential to introduce bias; however, the primary role of this individual was to identify and address any issues in stakeholder engagement. The director of engagement and the Alliance were seen as neutral parties/collaborators interested in seeing both academic and CBO stakeholders achieve their goals for the project. This was well known by all members of the team and established early in the project.

As results from the trial are being reviewed, the research team has had the opportunity to review and contribute to the analysis. While the peer-to-peer research project has concluded, the team will be disseminating the results of the research to each of the participating communities, paying attention to relevant communication channels for stakeholder groups. In addition, the team has submitted proposals for broader dissemination and replication of the program model in new communities. 
Corresponding Author: Emily R. Connors, M.S.; Clinical and Translational Science Institute Medical College of Wisconsin, Milwaukee, WI, USA (e-mail: connors.em83@gmail.com).

Funding Financial support for this project was provided by PCORI, CER 1310-07844.

\section{Compliance with Ethical Standards:}

Conflict of Interest: Emily Connors received consultation fees from the Alliance for Strong Families and Communities to analyze the qualitative data and support the development of this publication. All remaining authors declare that they do not have a conflict of interest.

\section{REFERENCES}

1. PCORI (2018a). About us. Retrieved August 28, 2018, from https://www. pcori.org/about-us

2. Ahmed S, Palermo A. Community engagement in research: frameworks for education and peer review. Am J Public Health., 2010;100(8), 1380.

3. PCORI (2018b). Public comments for PCORI's national priorities and research agenda. Retrieved August 28, 2018, from https://www.pcori. org/research-results/research-we-support/national-priorities-and-research-agenda/how-we-developed-our-0

4. Fleurence R, Selby JV, Odom-Walker K, Hunt G, Meltzer D, Slutsky $\mathbf{J R}$, Yancy $\mathbf{C}$. How the patient-centered outcomes research institute is engaging patients and others in shaping its research agenda. Health Affairs. 2013;32(2), 393-400.

5. Thompson VLS, Drake B, James AS, Norfolk M, Goodman M, Ashford L, Jackson S, Witherspoon M, Brewster M, Colditz G. A community coalition to address cancer disparities: transitions, successes and challenges. J Cancer Educ. 2014;30(4), 616-622.

6. Hasnain-Wynia R, Beal AC. Role of the patient-centered outcomes research institute in addressing disparities and engaging patients in clinical research. Clin Ther. 2014;36 (4), 619-623.

7. Selker HP, Wilkins $\mathbf{C H}$. From community engagement to communityengaged research, to broadly engaged team science. Transl Sci. 2017;1(1), 5-6.

8. Wallerstein $\mathbf{N}$, Duran B. Community-based participatory research contributions to intervention research: the intersection of science and practice to improve health equity. Am J Public Health. 2010;100 (Suppl 1), 40-46.
9. Ellis LE, Kass NE. How are PCORI-funded researchers engaging patients in research and what are the ethical implications? AJOB Empirical Bioethics. 2017;8 (1), 1-10.

10. Minkler, $\mathbf{M}$, Wallerstein, $\mathbf{N}$. The growing support for CBPR. In Minkler $\mathrm{M}$, Wallerstein $\mathrm{N}$ eds. Community-based participatory research for health: from process to outcomes (2nd, pp. 544) San Francisco: JosseyBass: 2008.

11. Gurke JG, Jones J, Yonas M, Guizzetti L, Virata M, Costlow M, Morton S, Elizabeth M. PCOR, CER, and CBPR: alphabet soup or complementary fields of health research?. Clin Transl Sci. 2013; 6 (6), 493-496.

12. PCORI. (2015a). What we mean by engagement. Retrieved August 28, 2018, from http://www.pcori.org/funding-opportunities/what-we-meanengagement

13. Ehlers AP, Davidson GH, Deeney K, Talan D. Methods for incorporating stakeholder engagement into clinical trial design. Generating Evidence \& Methods to improve patient outcomes (eGEMs), 2017;5(1), 1-7.

14. Goodman MS, Sanders Thompson VL. The science of stakeholder engagement in research: classification, implementation, and evaluation. Transl Behav Med.2017;7(3), 486-491.

15. Forsythe L, Heckert A, Margolis MK, Schrandt S, Frank L. Methods and impact of engagement in research, from theory to practice and back again: early findings from the Patient-Centered Outcomes Research Institute. Qual Life Res. 2018;27 (1), 17-31.

16. U.S. National Library of Medicine, ClinicalTrials.gov. Retrieved August 28, 2018, from https://www.clinicaltrials.gov/ct2/show/NCT02308696? term=peer+to+peer+support\&rank=3

17. Society of General Internal Medicine. Abstracts from the 35th annual meeting of the society of general internal medicine, 2012 Apr;27(Suppl 2):99-574

18. Glasser, B. The constant comparative method of qualitative analysis. grounded theory review: an international journal, 2008;7 (3).

19. PCORI. (2015b). PCORI engagement rubric. Retrieved August 28, 2018, from http://www.pcori.org/sites/default/files/Engagement-Rubric.pdf

20. Georgetown National Center for Cultural Competence. (2017). Cultural brokering. Retrieved August 28, 2018, from https://nccc.georgetown. edu/resources/brokering.html

21. Price A, Schroter S, Snow R, Hicks M, Harmston R, Staniszewska S, Parker S, Richards T. Frequency of reporting on patient and public involvement (PPI) in research studies published in general medical journal: a descriptive study. BMJ Open. 2018; 8(3).

22. Chi DL, Milgrom P, Gillette J. Engaging stakeholders in patientcentered outcomes research regarding school-based sealant programs. J Dent Hyg. 2018;92(1):16-22

23. Greene SM, Brandzel S, Wernli KJ. From principles to practice: realworld patient and stakeholder engagement in breast cancer research. Perm J. 2018;22:17-232. 